Int. J. Morphol.,

25(3):501-509, 2007.

\title{
Bases Anatómicas de la Gluteoplastía Dinámica
}

\author{
Anatomical Basis of the Dinamic Gluteoplaty
}

\section{"Laura Borgno; ** Varinia Scanniello; *** Omar Rompani; ${ }^{* * * * *}$ Alejandra Laxague \& ${ }^{* * * *}$ Sofía Páez}

BORGNO, L.; SCANNIELlO, V.; ROMPANI, O.; LAXAGUE, A. \& PAEZ, S. Bases anatómicas de la gluteoplastía dinámica Int. J. Morphol., 25(3):501-509, 2007.

RESUMEN: La disrupción anatómica del esfínter es el mecanismo más común de incontinencia fecal. La creación de un mecanismo esfinteriano con control voluntario permite mantener la contracción en el reposo o durante los movimientos y es uno de los principios fisiológicos y quirúrgicos para la continencia. Esta corrección puede realizarse mediante la transposición de músculo glúteo mayor alrededor del ano y recrear así un esfínter voluntario. La gluteoplastía dinámica consiste en la colocación de un marcapasos que permite la estimulación eléctrica crónica intermitente del músculo esquelético, resultando en la adaptación de la contractilidad basal. El objetivo del trabajo consiste en el estudio anatómico de la longitud in situ y esqueletizado del fascículo inferior del músculo glúteo máximo, de su principal pedículo nervioso, la altura en la cual penetra al músculo desde el cóccix, y la longitud de su rama más distal para finalmente, proponer variantes técnicas para este procedimiento. Se disecaron 21 regiones glúteas de ambos lados de cadáveres frescos y formolizados al 10\% de ambos sexos. La longitud del fascículo muscular sin esqueletizar osciló entre 17,8 y 19,65 cm, y esqueletizado entre 23,9 y $20.7 \mathrm{~cm}$. La distancia de penetración del nervio principal fue entre 11,25 10,2 cm y la del nervio más medial se situó promedialmente entre 8,72 y $5,97 \mathrm{~cm}$. La máxima longitud adquirida por el nervio principal hasta su rama más distal, osciló entre 10,4 y $9,2 \mathrm{~cm}$. El fascículo inferior posee una longitud final que permite rodear completamente al ano e incluso, llegar al isquion contralateral hechos que apoyan el uso de este músculo en las transposiciones para la corrección de las incontinencias anales, sin la necesidad de realizar abordajes desmedidos y lejanos, permitiendo a su vez, el uso de un músculo de la región, evitando tracciones del pedículo neurovascular y la consiguiente isquemia luego del procedimiento quirúrgico. Se proponen tres técnicas para la rotación del colgajo.

PALABRAS CLAVE: Gluteoplastía; Músculo glúteo máximo; Incontinencia anal.

\section{INTRODUCCIÓN}

La revisión histórica de los tratamientos realizados para el manejo de la incontinencia anal muestra que tanto los cirujanos generales, coloproctólogos, pediátricos y plásticos, así como las ramas afines a estas especialidades, han tomado un rol importante en su desarrollo.

Es justo resaltar que se han desarrollado múltiples técnicas, algunas con más o menos éxito, otras más o menos utilizadas, pero esta gran variedad responde muchas veces a que es necesario adaptar correctamente la técnica a la patología del paciente, de lo que se desprende que la selección de ambos es un paso fundamental en su manejo.
Tres mecanismos son necesarios para mantener la continencia fecal (Seidel et al., 1997): 1) Un reservorio, el recto; 2) Sensibilidad conservada, incluyendo la sensibilidad del canal anal y 3) Integridad funcional de los esfínteres anales interno y externo.

La disrupción anatómica (Enriquez Navascues \& Devesa-Mugica, 1994), del esfínter es el mecanismo más común de incontinencia fecal. Sin embargo, existen otras causas productoras de esta patología, entre las que se encuentran: la ablación por razones oncológicas, las malformaciones congénitas y los desórdenes neurológicos.

* Asistente del Departamento de Anatomía Humana Normal - Asistente de Clínica Quirúrgica “3”. Facultad de Medicina, Universidad de la República Oriental del Uruguay, Montevideo - Uruguay.

** Asistente del Departamento de Anatomía Humana Normal - Asistente del Departamento de Cirugía Plástica y Reparadora. Facultad de Medicina, Universidad de la República Oriental del Uruguay, Montevideo - Uruguay.

*** Ex Asistente del Departamento de Anatomía Humana Normal - Cirujano Coloproctólogo, Facultad de Medicina, Universidad de la República Oriental del Uruguay, Montevideo - Uruguay.

**** Ayudantes de Clase del Departamento de Anatomía Humana Normal, Facultad de Medicina, Universidad de la República Oriental del Uruguay, Montevideo - Uruguay. 
La recreación de un mecanismo esfinteriano con control voluntario permite mantener la contracción en el reposo o durante los movimientos y es uno de los principios fisiológicos y quirúrgicos para la continencia (Devesa et al., 1992). Idealmente se utiliza la esfinteroplastía o una técnica similar para su corrección. Cuando el esfínter anal está ausente, hipoplásico o dañado por sucesivas reparaciones, otras formas de corrección deben ser utilizadas.

La reconstrucción del esfínter externo del ano se ha realizado tradicionalmente mediante la transposición de músculo alrededor del ano, de forma tal de rodearlo y recrear un esfínter voluntario.

El músculo grácil ha sido el más frecuentemente utilizado, ya sea mediante transposición uni o bilateral según diferentes técnicas, la primera de ellas fue descrita por Pickrell et al. (1952). Los resultados han sido aceptables en algunos pacientes pero en la mayoría han sido insatisfactorios.

El músculo glúteo máximo se presenta como una alternativa al músculo grácil, al menos desde el punto de vista teórico, con múltiples ventajas respecto a otros músculos:

* Situación próxima al ano.

* Forma más natural de contracción.

* Actúa accesoriamente como músculo de la continencia.

* Es un músculo voluntario, potente y capaz de mantener tono basal.

* Su porción más inferior posee un pedículo neurovascular independiente que puede ser preservado cuando se disocian sus fibras y se liberan sus inserciones.

* Su desinserción y esqueletización permite rodear al ano con fibras musculares y no tendinosas.

* Si se suturan los sectores distales de ambos lados, uno con el otro, el efecto opuesto permite una mayor contracción con control voluntario.

La gluteoplastía fue descrita a principio del siglo XX, concretamente en 1902, por Chetwood. En 1909 Schoemaker describió un método similar de trasposición de músculo glúteo, utilizando sus porciones proximales de forma bilateral.

La utilización del músculo glúteo máximo ha ganado renovado interés en la década de los 80 , donde su uso ha mostrado resultados aceptables, la cual consiste en trasponer el fascículo inferior del músculo hacia el lado opuesto primero pasando por el sector posterior y luego rodeando al sector terminal del recto por su sector anterior, manteniendo la inserción del mismo a nivel sacrococcígeo.
A partir de la década del 80 se han introducido varias modificaciones a la técnica original de Chetwood, Stone (1929) desarrolla el uso del músculo glúteo máximo para la reparación del esfínter anal, deslizándolo alrededor del ano, actuando a nivel del canal anal indirectamente a través de colgajos fasciales.

Biström (1944) describió una técnica que parece ser más racional que la de Chetwood debido a que se adapta más cercanamente a los principios de preservación neurovascular y permite mayor longitud muscular para la sutura. Esta técnica consiste en mantener las inserciones distales del fascículo inferior, desinsertando sus amarres sacrococcígeos y realizar, a $2 \mathrm{~cm}$ de este borde, un orifico en dirección de las fibras de ambos colgajos, entonces ambos son suturados uno sobre el otro, superpuestos y el orificio anal, previamente desinsertado, es posicionado entre ambos orificios, suturando el músculo a la piel perianal.

No obstante, la técnica no es fácil de llevar a cabo y se corre el riesgo de producir isquemia e infección de la brecha perianal.

En 1979, Prochiantz (1979) describió una técnica que sugiere algunas modificaciones a la originalmente descrita por Schoemaker, aplicándola cuando no es posible rodear completamente el ano. Este autor sugiere adicionar una segunda angulación al intestino, creando un anillo con músculo glúteo por encima del músculo elevador del ano dirigiéndolo hacia el lado opuesto, determinando un cambio en la dirección del ángulo anorrectal.

En 1981, Bruining et al. describieron una técnica que utiliza la trasposición de los sectores proximales del músculo glúteo máximo. En 1982 Hentz, publica otra técnica de gluteoplastía que es una modificación de la de Bistrom pero con una ventaja sustancial, no desinserta el ano y se realiza mediante 2 incisiones independientes perianales, los dos extremos de cada lado son pasados alrededor del recto al lado contralateral, uno anterior y otro posteriomente y suturados juntos, pero teniendo el inconveniente y la dificultad de rodear al ano. Devesa \& Fernández (1997) señalaron que, luego de un período inicial de entrenamiento, el músculo glúteo máximo parece mantener en reposo el tono suficiente para evitar el escape de gases. Esta técnica ha tenido gran aceptación y ha sido reproducida y modificada mínimamente por diferentes autores, como Pearl, Devesa et al. (1991)y Christiansen et al., 1992.

Chen \& Zhang (1987) describieron una modificación al procedimiento de Schoemakers (1909) quien posicionaba uno de los colgajos por delante y otro por detrás del ano, fijándolos al isquion contralateral y suturándolos uno con el otro. 
Al igual que Prochiantz \& Gross (1982), Devesa \& Fernández (1997) resaltaron los efectos beneficiosos de la plastía en la continencia y aducen que ésta se debe no sólo a que las fibras se suturan en dirección cruzada lo que produce un cierre sustancioso del conducto rectoanal sino a que esta técnica aumenta y acentúa el ángulo anorectal.

Finalmente, en 1992 Devesa et al., publicaron un nuevo procedimiento de gluteoplastía. Este método fue diseñado para evitar tensión excesiva de la plastía en algunos casos o debido a la imposibilidad técnica de poder rodear completamente al recto, como es mencionado en otras técnicas. En este caso, un solo sector terminal del músculo no importando cual, es dividido y cruzado al sector contralateral. El colgajo que no es cruzado, completa el anillo perianal, en caso que los sectores terminales cruzados no logren hacerlo. Los músculos opuestos creados, actúan como un esfínter o valva con el sector distal del recto. Esta técnica ha sido completada en 11 de 25 pacientes con la implantación sincrónica de un estimulador.

Es sabido que la estimulación eléctrica crónica intermitente del músculo esquelético resulta en la adaptación de la contractilidad, y las propiedades metabólicas e histoquímicas de las fibras musculares. La estimulación muscular intermitente luego de la trasposición muscular fue introducida por Carpentier \& Chachques (1985) para la realización de la cardiomioplastía dinámica.

En 1996 Guelinckx et al., realizaron 11 gluteoplastías como procedimiento de sustitución anal y en 4 de ellas se realizó la gluteoplastía dinámica. La primera gluteoplastía con implantación de electrodos y generadores de pulsos fue realizada en 1992.

La gluteoplastía dinámica aparece en el momento actual, como una técnica esperanzadora en el manejo y tratamiento de las incontinencias anales graves, y su desarrollo se está llevando a cabo en los centros especializados en el manejo de esta patología.

Los resultados en el uso de la trasposición glútea para la incontinencia anal en las diferentes series, son bastante similares a los descritos cuando se utiliza el músculo grácil sin estimulador.

Pearl et al., refieren que luego de 3 meses de realizado el procedimiento, la continencia para sólidos se vio en 6 de 7 pacientes, manteniendo esos pacientes la sensibilidad y el control en el pasaje de materias líquidas, presentando un solo paciente continencia total incluso para gases. Los resultados fueron juzgados por los pacientes como buenos. No existieron muertes asociadas al procedimiento, pero sí infecciones de la herida operatoria en el $43 \%$ de los casos; sin embargo ninguno requirió colostomía de transitación por sepsis.

Devesa et al. destacan la dificultad para determinar, en el postoperatorio inmediato, la evaluación clínica de la continencia. Sin embargo, con el correr de los días aparece el control para las materias formadas llegando, en algunos casos, a la normalidad en la continencia. No existieron pacientes que presentaran un empeoramiento de su incontinencia. La electromiografía mostró actividad espontánea en el músculo y los resultados electromiográficos siempre fueron superiores a los preoperatorios.

Christiansen et al., mostraron resultados inferiores a los reportados por Pearl et al. y por Devesa et al., destacan que no se presentaron cambios en relación a la sensibilidad rectal y la capacidad de continencia no cambió en el período postoperatorio.

De los trabajos consultados surge la dificultad para establecer un estado mensurable de incontinencia, probablemente debida a varias causas, entre las que se destacan:

- Variación en el criterio de selección de los pacientes.

- Diferentes movimientos del intestino para materias líquidas o sólidas.

- Performance técnica deficiente.

- Complicaciones postoperatorias.

Esto determina la diferente evaluación de los resultados y hace verdaderamente difícil comparar tanto las técnicas como los resultados.

Bases anatómicas. El músculo glúteo máximo es el más voluminoso de los músculos glúteos (Latarjet \& Ruiz Liard, 1989), se origina de la cara glútea del hueso coxal a nivel de la línea glútea posterior del ilion y del área rugosa del hueso incluyendo la cresta ilíaca, del sacro y del cóccix, así como de las fascia que recubre el músculo glúteo medio y del ligamento sacrotuberal.

Las inserciones se realizan a nivel del tracto iliotibial de la fascia lata, donde se fusiona con el tendón del músculo tensor de la fascia lata y en la tuberosidad glútea entre los músculos vasto lateral y aductor magno.

Su inervación está dada por el nervio glúteo inferior, que se origina del plexo sacro (L5, S1, S2), penetrando al músculo por su cara más profunda. La vascularización está dada por la arterias glúteas superior e inferior. 
El tercio inferior del músculo glúteo máximo puede ser removido sin detrimento de la estabilidad pelviana o compromiso funcional del resto de los fascículos. Las fibras paralelas son alargadas lo que las hace ideales para ser utilizadas en las transposiciones o reconstrucciones.

Guelinckx et al. en un estudio con 10 cadáveres identificaron el patrón de vascularización e inervación del músculo glúteo máximo. El pedículo neurovascular para este autor, está localizado cerca del origen del músculo; sin embargo no refieren a cuantos centímetros. Señalan que no existe tracción en la arteria glútea inferior, su vena o nervio si éste es seccionado distalmente y se utiliza para rodear al ano.

Los objetivos del trabajo consisten en el estudio anatómico detallado de la porción inferior del músculo máximo, su longitud in situ y luego de esqueletizado, así como el estudio de su principal pedículo nervioso y la altura en la cual penetra al músculo desde el cóccix, sus ramas de división y distribución.

El propósito de esta invesigación es determinar las características generales del fascículo inferior del músculo glúteo máximo con el fin de realizar la trasposición muscular en la gluteoplastía dinámica para el tratamiento de las incontinencias anales, de forma tal de determinar el sitio correcto de colocación del estimulador y conocer la posibilidad de rodear completamente al ano en la realización de la técnica quirúrgica. Por último, se proponen variantes técnicas para la rotación del colgajo y la realización de la gluteoplastía dinámica.

\section{MATERIAL Y MÉTODO}

Se disecaron 21 regiones glúteas derechas e izquierdas de cadáveres de ambos sexos ( 11 femeninos y 10 masculinos) de los cuales 11 eran frescos y 10 formolizados, estos últimos según técnica del Departamento y Cátedra de Anatomía de la Facultad de Medicina de Montevideo, Uruguay.

Mediante abordaje cutáneo, siguiendo una línea trazada desde la unión sacro coccígea a la inserción trocantereana del músculo glúteo máximo, pasando por el pliegue glúteo; se procedió a la exposición del borde inferior y cara superficial del músculo glúteo máximo y luego, a la visualización del fascículo inferior, disecando su intersticio muscular. En dos casos fue necesario el labrado de dicho fascículo debido a que fue prácticamente imposible su reconocimiento.
A ese nivel se realizó la disección de los pedículos neurovasculares y la identificación de ramas musculares nerviosas accesorias procedentes del plexo sacro.

Se identificaron los nervios cutáneo posterior del muslo, glúteo inferior y pudendo, así como los elementos vasculares de la región.

Se efectuaron las siguientes mediciones:

- Longitud del fascículo muscular, desde su origen sacrococcígea a la fascio-trocantereana in situ a nivel del borde inferior del mismo.

- Distancia de penetración del nervio principal, desde el cóccix hasta el sitio de proyección en el borde superior del fascículo.

- Porcentaje de existencia de pedículos nerviosos accesorios y sus longitudes de penetración, tomando como reparo el cóccix.

Una vez realizadas dichas medidas, se procedió a la esqueletización del músculo desinsertándolo del trocánter y liberándolo hasta el sacro-cóccix, disecando las ramas de división de su pedículo nervioso por su cara profunda esqueletizándolo hasta sus sectores más distales, midiéndose las longitudes del fascículo esqueletizado y del nervio hasta su ramo más distal.

\section{RESULTADOS}

Los resultados se expresan en las Tablas I y II y los promedios se pueden resumir de la siguiente forma:

Cadáveres frescos:

- Longitud del fascículo muscular sin esqueletizar desde su origen a su inserción: $15 \mathrm{~cm}-24,8 \mathrm{~cm}$ con una media de 17,8 $\mathrm{cm}(\mathrm{n}=11)$.

- Longitud del fascículo muscular esqueletizado desde su origen a su inserción: $21,2 \mathrm{~cm}-28 \mathrm{~cm}$ con una media de $23,9 \mathrm{~cm}$ $(n=11)$.

- Distancia de penetración del nervio principal del fascículo muscular desde el cóccix al borde superior: $9 \mathrm{~cm}$ - 13,8 cm con una media de $11,25 \mathrm{~cm}(\mathrm{n}=11)$.

- Longitud del nervio principal desde el borde inferior del músculo glúteo máximo a su ramo más distal: 8 cm-16,6 cm con una media de $10,4 \mathrm{~cm}(\mathrm{n}=11)$.

- Distancia de penetración del nervio más medial, colateral del plexo sacro, accesorio del fascículo inferior del músculo glúteo máximo desde el cóccix al borde superior: $6,5 \mathrm{~cm}$ $10,5 \mathrm{~cm}$ con una media de $8,72 \mathrm{~cm}(\mathrm{n}=10)$. 
Tabla I. Longitud del fascículo muscular del m. glúteo máximo y medidas del nervio glúteo inferior en cadáveres frescos de ambos sexos.

\begin{tabular}{|c|c|c|c|c|c|c|c|c|c|c|c|}
\hline \multirow[b]{2}{*}{ LADO } & \multicolumn{2}{|c|}{$\begin{array}{c}1 \\
\text { Hombre }\end{array}$} & \multicolumn{2}{|c|}{$\begin{array}{c}2 \\
\text { Mujer }\end{array}$} & \multicolumn{2}{|c|}{$\begin{array}{c}3 \\
\text { Mujer }\end{array}$} & \multicolumn{2}{|c|}{$\begin{array}{c}4 \\
\text { Mujer }\end{array}$} & \multicolumn{2}{|c|}{$\begin{array}{c}5 \\
\text { Hombre }\end{array}$} & \multirow{2}{*}{$\begin{array}{c}\mathbf{6} \\
\text { Mujer } \\
\text { I } \\
\end{array}$} \\
\hline & D & I & D & I & D & I & D & I & D & I & \\
\hline Longitud fascículo sin esqueletizar (cm) & 16 & 16 & 16 & 18 & 20 & 18,5 & 15,2 & 17,5 & 19.8 & 24,8 & 15,0 \\
\hline Longitud fascículo esque letizado (cm) & 25 & 27 & 23 & 23 & 22 & 21.5 & 21,2 & 23,5 & 27.8 & 28,0 & 21,4 \\
\hline $\begin{array}{l}\text { Distancia de penetración del nervio desde el } \\
\text { cóccix }(\mathrm{cm})\end{array}$ & 9 & 11 & 10,5 & 11 & 12 & 10 & 11,4 & 11,5 & 11.6 & 13,8 & 12,5 \\
\hline Longitud del nervio principal $(\mathrm{cm})$ & 9 & 11 & 9 & 8.5 & 8 & 9 & 12,2 & 8,5 & 12.6 & 16,6 & 10,5 \\
\hline $\begin{array}{l}\text { Distancia desde el cóccix al nervio más } \\
\text { medial }(\mathrm{cm})\end{array}$ & 7 & 6,5 & 8 & 7 & 10 & 8.5 & 10,5 & 10,2 & 10,0 & 10,0 & $\mathrm{~N} / \mathrm{P}$ \\
\hline
\end{tabular}

Cadáveres formolizados:

- Longitud del fascículo muscular sin esqueletizar desde su origen a su inserción: $17 \mathrm{~cm}-23 \mathrm{~cm}$ con una media de 19,65 cm. $(n=10)$.

- Longitud del fascículo muscular esqueletizado desde su origen a su inserción: $18 \mathrm{~cm}-23,5 \mathrm{~cm}$ con una media de $20,7 \mathrm{~cm} \mathrm{(n=10).}$

- Distancia de penetración del nervio principal del fascículo muscular desde el cóccix al borde superior: $4 \mathrm{~cm}-15 \mathrm{~cm}$ con una media de $10,2 \mathrm{~cm}(\mathrm{n}=10)$.

- Longitud del nervio principal desde el borde inferior del músculo glúteo máximo a su ramo más distal: $6.5 \mathrm{~cm}-12$ $\mathrm{cm}$ con una media de $9,2 \mathrm{~cm}(\mathrm{n}=10)$.

- Distancia de penetración del nervio más medial, colateral del plexo sacro, accesorio del fascículo inferior del músculo glúteo máximo desde el cóccix al borde superior: $5.5 \mathrm{~cm}$ $6,6 \mathrm{~cm}$ con una media de $5,97 \mathrm{~cm}(\mathrm{n}=4)$.
Es de destacar la variación que existe entre los cadáveres frescos y los formolizados que puede ser fácilmente explicada por la retracción muscular que sufre la pieza formolizada, lo que implica que en el análisis de los resultados es francamente demostrable la menor longitud del fascículo muscular una vez esqueletizado; sin embargo, posee mayor longitud in situ que el fresco.

Las diferencias entre ambos fascículos, antes y después de su disección, son notables, teniendo variaciones para el fresco de $6,1 \mathrm{~cm}$ y para el formolizado de $1,05 \mathrm{~cm}$.

La distancia de penetración del nervio principal y su longitud hasta la rama más distal mantienen relación en ambos tipos de piezas con promedios que oscilan entre 10,2 $\mathrm{cm}$ y $11,25 \mathrm{~cm}$ para los formolados y los frescos en cuanto a su distancia de penetración, y de $9,2 \mathrm{~cm}$ y $10,4 \mathrm{~cm}$ en lo que respecta a su esqueletización, respectivamente.

El nervio más medial correspondió a un ramo del plexo sacro, que la gran mayoría de las veces brindó ramos

Tabla II. Longitud del fascículo muscular del m. glúteo máximo y medidas del nervio glúteo inferior en cadáveres formolizados de ambos sexos.

\begin{tabular}{|c|c|c|c|c|c|c|c|c|c|c|}
\hline \multirow[b]{3}{*}{ LADO } & \multirow{2}{*}{\multicolumn{2}{|c|}{$\begin{array}{c}7 \\
\text { Mujer }\end{array}$}} & \multirow{2}{*}{\multicolumn{2}{|c|}{$\begin{array}{c}8 \\
\text { Hombre }\end{array}$}} & \multirow{2}{*}{\multicolumn{2}{|c|}{$\begin{array}{c}9 \\
\text { Hombre }\end{array}$}} & \multirow{2}{*}{\multicolumn{2}{|c|}{$\begin{array}{c}10 \\
\text { Mujer }\end{array}$}} & \multirow{2}{*}{\multicolumn{2}{|c|}{$\begin{array}{c}11 \\
\text { Hombre }\end{array}$}} \\
\hline & & & & & & & & & & \\
\hline & D & $\mathbf{I}$ & D & $\mathbf{I}$ & D & $\mathbf{I}$ & D & $\mathbf{I}$ & D & $\mathbf{I}$ \\
\hline Longitud fascículo sin esqueletizar (cm) & 17 & 17 & 21,5 & 23 & 19 & 18 & 18 & 19.5 & 22,0 & 22 \\
\hline Longitud fascículo esqueletizado (cm) & 20 & 18 & 23,0 & 22 & 19 & 19 & 18.5 & 21 & 23,5 & 23,5 \\
\hline $\begin{array}{l}\text { Distancia de penetración del nervio desde } \\
\text { el cóccix }(\mathrm{cm})\end{array}$ & 10 & 15 & 12,5 & 9.5 & 11 & 9,5 & 10 & 4 & 11,0 & 10 \\
\hline Longitud del nervio principal $(\mathrm{cm})$ & 12 & 9.6 & 8,0 & 8 & 9,5 & 8 & 6,5 & 9 & 10,5 & 11 \\
\hline $\begin{array}{l}\text { Distancia desde el cóccix al nervio más } \\
\text { medial }(\mathrm{cm})\end{array}$ & 7 & 5.5 & 6,0 & 5.8 & $\mathrm{~N} / \mathrm{P}$ & N/P & $\mathrm{N} / \mathrm{P}$ & $\mathrm{N} / \mathrm{P}$ & N/P & $\mathrm{N} / \mathrm{P}$ \\
\hline
\end{tabular}


para el sector más medial y otras veces se agotó en la región sacra a nivel de los ligamentos y fascias locales.

Habitualmente, el nervio glúteo inferior, se dividió en 2 o 3 ramos terminales, que a su vez, se subdividieron en 2 ramos.

El ramo terminal más lateral y sus terminales, en general, se relacionan y transitan por el borde inferior del fascículo muscular hasta acercarse a la inserción trocanteriana del músculo glúteo máximo.

El ramo más medial se distribuye en la mitad medial del músculo glúteo máximo y, en general, no llega al borde inferior.

En una disección de ambas regiones glúteas se encontró en el lado derecho lo que parecía la existencia de dos nervios principales; sin embargo, al efectuar la disección en sentido proximal se encontró un origen de división alto del nervio glúteo inferior.

\section{DISCUSIÓN}

Existen pocas referencias anatómicas que permitan determinar el sitio exacto de hallazgo del nervio glúteo inferior, no obstante se reconoce como poco variable.

Usualmente, éste se encuentra localizado a una distancia de $8 \mathrm{~cm}$ distal al borde del sacro, penetrando la superficie profunda del músculo luego de dejar la escotadura ciática mayor por debajo del músculo piramidal (Devesa \& Fernandez, 1997)

Algunos autores aducen que a veces pueden observarse uno o dos pedículos neurovasculares secundarios muy próximos al sacro, hecho que puede determinar una limitación en la movilización del colgajo. (Devesa \& Fernandez).

Si bien estas ramas secundarias existen casi constantemente, hemos observado que el ramo nervioso secundario más medial del nervio glúteo inferior hallado, se distribuye en el músculo en sus porciones más superiores no llegando al borde inferior del mismo y la rama más medial colateral del plexo sacro llega en los cadáveres frescos hasta los $8,7 \mathrm{~cm}$, más allá de la distancia de penetración del nervio principal encontrada por los mismos autores, lo que no supondría dificultad alguna en la rotación del colgajo in vivo, ya que una vez esqueletizado el músculo, la distancia desde sus inserciones mediales a la proximidad anal, no son mayores que ésta.
Por otro lado, se ha constatado una longitud por demás aceptable del propio fasículo inferior que varía entre los 20 y $23 \mathrm{~cm}$ en cadáveres formolizados y frescos, respectivamente.

Por todo lo expuesto, en relación al trabajo anatómico se concluye que en el músculo fresco una vez esqueletizado el fascículo inferior, su longitud final permite rodear completamente al ano e incluso, llegar al isquion contralateral, hechos que apoyan el uso de este músculo en las transposiciones para la corrección de las incontinencias anales, sin la necesidad de realizar abordajes desmedidos y lejanos, permitiendo, a su vez, el uso de un músculo de la región, evitando tracciones del pedículo neurovascular y la consiguiente isquemia luego del procedimiento quirúrgico.

La realización de una gluteoplastía requiere tener en cuenta preceptos quirúrgicos básicos:

1. El equipo técnico: el cirujano colorectal, experto en cirugía anal, el cirujano plástico encargado de la rotación y la trasposición de colgajos y los técnicos expertos en el manejo de los estimuladores.

2. Un colgajo muscular indemne desde el punto de vista de sus vascularización e inervación y lo suficientemente largo como para permitir la confección del neoesfínter.

3. Una incisión que asegure la correcta disección del fascículo inferior del músculo glúteo máximo, con mínimas consecuencias funcionales y estéticas, evitando en lo posible la disección subcutánea. (Fig. 1).

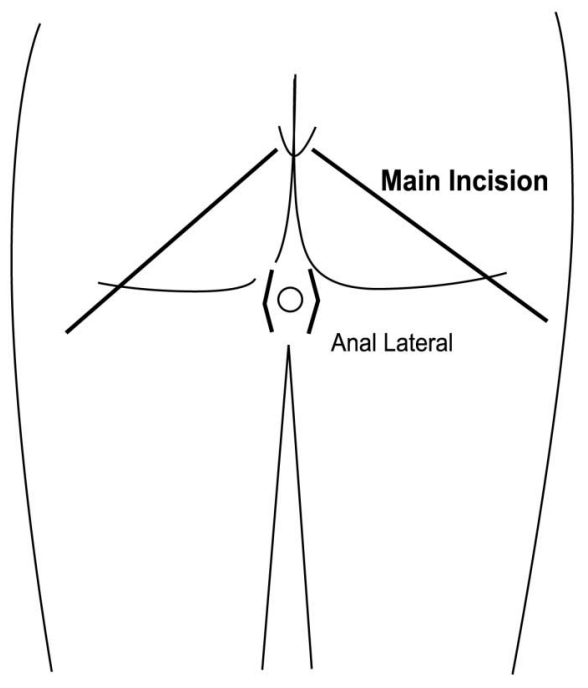

Fig. 1. Incisiones cutáneas interna y externa para la confección y trasposición del colgajo pediculado. 
4. Seleccionar adecuadamente a los candidatos para la aplicación de la técnica, siendo aquellos pacientes portadores de incontinencias anales en los que no es posible la reparación directa del esfínter.

\section{Realización del túnel perianal.}

6. La necesidad de sumar una segunda angulación al sector rectoanal.

7. La confección del loop, siendo fundamental, en este caso, evitar el retorno a pasos previos.

El análisis de los resultados de los autores consultados, muestra que la gluteoplastía ideal aún no ha sido diseñada, ya que quedan algunas interrogantes por resolver. entre las que se destacan: la de determinar qué inserción del fascículo inferior debe ser desinsertada y rotada de forma tal de que sea menos dificultoso técnicamente, la necesidad o no de desinsertar el ano y el pasaje del colgajo por el sector anterior a nivel del centro fibroso del periné o por el rafe anal posterior.

A la luz de estas consideraciones y de acuerdo a los datos anatómicos obtenidos, tanto del músculo como de su pedículo vasculonervioso, es que nos permitimos proponer teóricamente 3 tipos diferentes de loop a realizar con músculo glúteo máximo.

A. Loop en L bilateral: Consiste en un colgajo muscular que se toma del isquion contralateral y se sutura a las fascias y tendones de los músculos isquiosurales. La acción del loop permite llevar el ano en sentido posterior, oponiéndose a los movimientos del músculo puborectal cuando éste no está dañado. (Fig. 2).

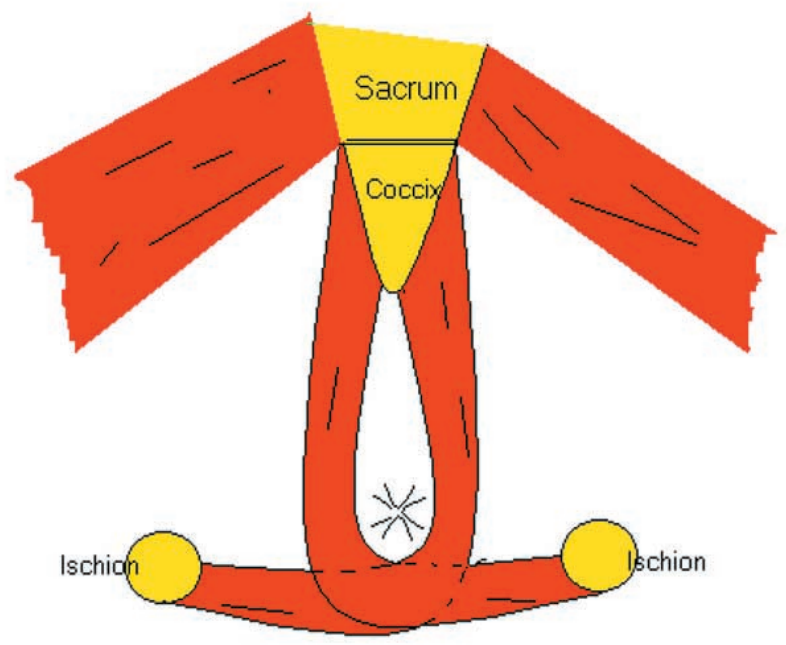

Fig. 2. Loop en L bilateral.
B. Loop en forma de gota: Cuando el fascículo muscular no llega al isquion contralateral, ambos fascículos se suturan superponiéndose o no, dependiendo de la delgadez del fascículo dándole forma de gota. (Fig. 3).

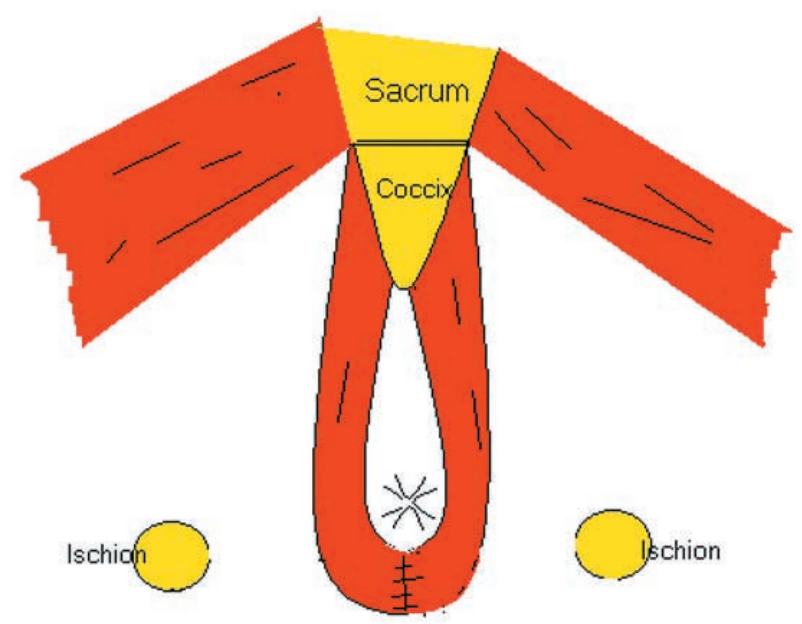

Fig. 3. Loop en forma de gota.

C. Loop en forma de 6, a izquierda, o d, a derecha: Cuando uno de los fascículos no puede ser usado para rodear al ano, el contralateral puede ser colocado por detrás del mismo, en forma de 6 o de d, dependiendo del lado utilizado. (Fig. 4).

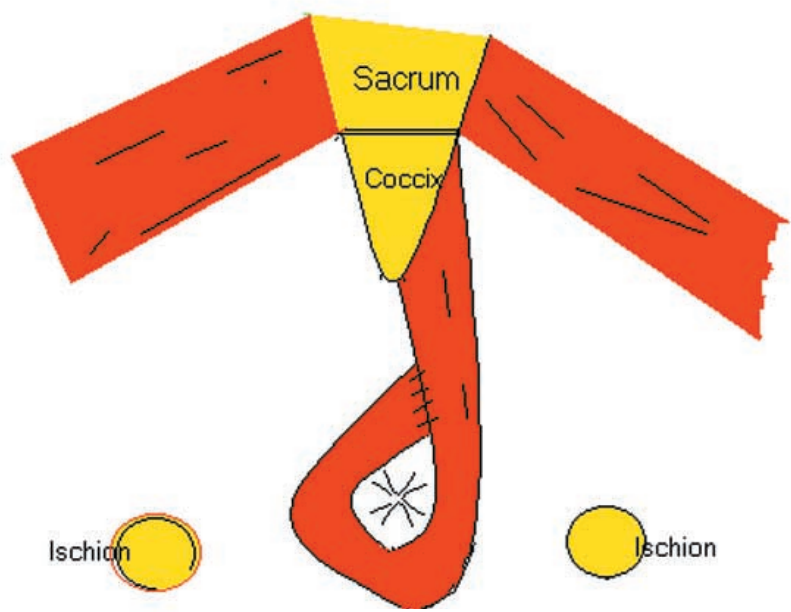

Fig. 4. Loop en forma de 6 o d. 
D. Implantación del esfínter anal artificial $A B S ®$ : que es una modificación técnica del esfínter urinario artificial (AMS 800®), efectuada expresamente para su implantación perianal. Se trata de una prótesis totalmente implantable de elastómero de silicona sólido consistente en tres componentes interconectados entre sí por 2 tubos: un manguito oclusivo, un balón regulador de presión y una bomba de control. Con este dispositivo, el paciente es capaz de regular la apertura y el cierre del esfínter manipulando la bomba de control.

Concluimos que el fascículo inferior posee una longitud final que permite rodear completamente al ano e incluso llegar al isquion contralateral, hechos que apoyan el uso de este músculo en las transposiciones para la corrección de las incontinencias anales, sin la necesidad de realizar abordajes desmedidos y lejanos, permitiendo a su vez el uso de un músculo de la región, evitando tracciones del pedículo neurovascular y la consiguiente isquemia luego del procedimiento quirúrgico.

Si bien el dispositivo eléctrico mencionado es una alternativa adecuada para tratar a pacientes con incontinencia fecal cuando otras alternativas han fracasado, su indicación debe ser cautelosa hasta el momento en que la experiencia en la técnica asegure una mayor tasa de éxitos que de fracasos.

BORGNO, L.; SCANNIELLO, V.; ROMPANI, O.; LAXAGUE, A. \& PAEZ, S. Anatomical basis of dynamic gluteoplasty. Int. J. Morphol., 25(3):501-509, 2007.

SUMMARY: The anatomical disruption of the sphincter is the most common mechanism of fecal incontinence. The creation of an sphincter mechanism with voluntary control allows to maintain the contraction during rest or movements and is one of the physiological and surgical principles of continence. This correction can be made by the gluteus maximus muscle transposition around the anus recreating a voluntary sphincter. The dynamic gluteoplasty consists in the positioning of a nerve stimulator that allows the intermittent chronic electrical stimulation of the skeletal muscle resulting in the adaptation of the basal contractility. The objective of the work is the anatomic study of the inferior fascicle of the gluteus maximus muscle (its length in situ and squeletized) and of its main nervous pedicle (the height in which penetrates the muscle from coccyx, and the length of its distal branch) so as to propose technical changes for the procedure. Twenty and one gluteus regions of both sides from fresh and formalized cadavers of both sexes were dissected. The length of muscular fascicle without squeletized oscillated between 17.8 and $19.65 \mathrm{~cm}$, and squeletized between 23.9 and $20.7 \mathrm{~cm}$. The distance of penetration of the main nerve was between 11.25 and $10.2 \mathrm{~cm}$ and the one of the most internal nerve was located promedially between 8.72 and 5.97 $\mathrm{cm}$. The maximun length acquired by the main nerve until its distal branch oscillated between 10.4 and $9.2 \mathrm{~cm}$. The inferior fascicle has a final length that allows to surround completely the anus or even to reach the contralateral isquion, facts which support the use of this muscle in the transpositions for the correction of the anal incontinence, without making excessive or distant boardings, allowing as well the use of a muscle from the region, avoiding tractions of neurovascular pedicle and the consequent isquemia after the surgical procedure. Three technical changes for the rotation of flap are proposed.

KEY WORDS: Gluteoplasty; Maximus gluteus muscle; Anal Incontinence.

\section{REFERENCIAS BIBLIOGRÁFICAS}

Biström, O. Plastiches Erzatz des M. Sphincter Ani. Acta Chir.Scan., 90:431, 1944.

Bruining, H. A.; Bos, K. E.; Colthoff, E. G. \& Tolhurst, D. E. Creation of an anal sphincter mechanism by bilateral proximally based gluteal muscle transposition. Plas.Reconst. Surg., 67:70-2, 1981.

Carpentier, A. \& Chachques, J. C. Myocardial substitution with stimulated skeletal muscle: First succesful clinical case. Lancet, 1:1267-1270, 1985.

Chen, Y. L. \& Zhang, X. H. Reconstruction of rectal sphincter by transposition of gluteus maximus muscle for fecal incontinence. J. Pediatr. Surg., 22:62-4, 1987.
Chetwood, C. H. Plastic Operation for Restoration of the Sphincter Ani with Report of a Case. Med. Rec. Ann., 61:529-31, 1902.

Christiansen, J.; Ronholt Hansen; C. \& Rasmussen, O. Bilateral gluteus maximus transposition for anal incontinence. Br. J. Surg., 82: 903-5, 1995.

Devesa, J. M.; Vicente, E.; Enríquez, J. M.; Nuño, J.; Bucheli, P.; de Blas, G. \& Villanueva, M. G. Total fecal incontinence- A new method of gluteus maximus transposition: preliminary results and report of previous experience withsimilar procedures. Dis. Colon Rectum, 35(4):339-49, 1992. 
Devesa, J. \& Fernández, J. Bilateral Gluteoplsty for anal incontinence. Sem. Colon and Rectal Surg., 8(2): 1039, 1997.

Enriquez Navascues, J. M. \& Devesa-Mugica, J. M. Traumatic anal incontinence: Rol of unilateral Gluteus Maximus Transposition Supplementing and supporting Direct Anal Sphincteroplasty. Dis. Colon Rectum, 37(8): 766-9, 1994.

Guelinckx, P. J.; Sinsel, N. K. \& Gruwez, J. A. Anal sphincter reconstruction with the gluteus maximus muscle: anatomic and physiologic considerations concerning conventional and dynamic gluteoplsty. Plast. Reconst. Surg., 98(2):293-302, 1996.

Hentz, V. R. Construction of a rectal sphincter using the origin of the gluteus maximus muscle. Plast. Reconst. Surg., 70: 82-5, 1982 .

Latarjet, M. \& Ruiz Liard, A. In: Anatomía Humana. $2^{\mathrm{a}}$ ed. México, Panamericana, 1989. pp. 785-7.

Pearl, R. K.; Prasad, M. L.; Nelson, R. L.; Orsay, C. P. \& Abcarian, H. Bilateral gluteus maximus transposition for anal incontinence. Dis. Colon Rectum, 34(6):478-81, 1991.

Pickrell, K. L.; Broadbent, T. R.; Masters F. W. \& Metzger, J. T. Construction of rectal sphincter and restoration of anal continence by transplanting the gracilis muscle: A report of four cases in children. Ann. Surg., 135:853-62, 1952.

Prochiantz, A. Myoplastie fessiére á visée sphinctérienne, pour le traitement des incontinences fécales des agénésies sacrées et des malformations anorectales opérées. Chir. Pédiatr., 20:63-7, 1979.
Prochiantz, A. \& Gross, P. Gluteal myoplasty for sphincter replacement. Principles, results and prospects. J. Pediatr. Surg., 17:25-30, 1982.

Schoemaker, J. Un nouveau procédé opératoire por la reconstitution du sphincter anal. Sem. Med., 29:160-3, 1909.

Seidel, S. P.; Georgeson, K. E. \& Vasconez, L. O. Gluteus Maximus Transposition Flap for Sphintcter Reconstruction. In Grabb's Encyclopedia of Flaps. B. Strauch, L. O. Vasconez, and E. J. Hall-Findlay, eds. $2^{\text {nd. }}$ Ed. Lippincot-Raven, Philadelphia. 1599-1602; 1997.

Stone, H. B. Plastic operation for anal incontinence. Arch. Surg., 18:845-51, 1929.

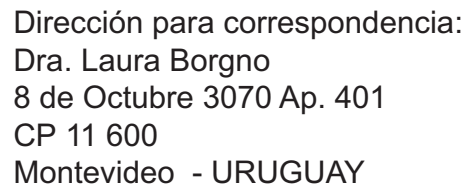

Dirección para correspondencia:

Dra. Laura Borgno

8 de Octubre 3070 Ap. 401

CP 11600

Montevideo - URUGUAY

Email: Imborgno@hotmail.com

Recibido : 10-10-2006

Aceptado: 23-04-2007 
